Review

\title{
Control of Organ Abscission and Other Cell Separation Processes by Evolutionary Conserved Peptide Signaling
}

\author{
Chun-Lin Shi ${ }^{\mathbb{D}}$, Renate Marie Alling, Marta Hammerstad and Reidunn B. Aalen * $\mathbb{D}$ \\ Section for Genetics and Evolutionary Biology, Department of Biosciences, University of Oslo, \\ N-0316 Oslo, Norway \\ * Correspondence: reidunn.aalen@ibv.uio.no; Tel.: +47-48218985
}

Received: 6 June 2019; Accepted: 10 July 2019; Published: 15 July 2019

check for updates

\begin{abstract}
Plants both generate and shed organs throughout their lifetime. Cell separation is in function during opening of anthers to release pollen; floral organs are detached after pollination when they have served their purpose; unfertilized flowers are shed; fruits and seeds are abscised from the mother plant to secure the propagation of new generations. Organ abscission takes place in specialized abscission zone (AZ) cells where the middle lamella between adjacent cell files is broken down. The plant hormone ethylene has a well-documented promoting effect on abscission, but mutation in ethylene receptor genes in Arabidopsis thaliana only delays the abscission process. Microarray and RNA sequencing have identified a large number of genes differentially expressed in the AZs, especially genes encoding enzymes involved in cell wall remodelling and disassembly. Mutations in such genes rarely give a phenotype, most likely due to functional redundancy. In contrast, mutation in the INFLORESCENCE DEFICIENT IN ABSCISSION (IDA) blocks floral organ abscission in Arabidopsis. $I D A$ encodes a small peptide that signals through the leucine-rich repeat receptor-like kinases HAESA (HAE) and HAE-LIKE2 (HSL2) to control floral organ abscission and facilitate lateral root emergence. Untimely abscission is a severe problem in many crops, and in a more applied perspective, it is of interest to investigate whether IDA-HAE/HSL2 is involved in other cell separation processes and other species. Genes encoding IDA and HSL2 orthologues have been identified in all orders of flowering plants. Angiosperms have had enormous success, with species adapted to all kinds of environments, adaptations which include variation with respect to which organs they shed. Here we review, from an evolutionary perspective, the properties of the IDA-HAE/HSL2 signaling module and the evidence for its hypothesized involvement in various cell separation processes in angiosperms.
\end{abstract}

Keywords: IDA/IDL; abscission; cell separation; peptide signaling; abscission zone

\section{Introduction}

Cell separation processes, especially abscission, are in general of great importance in plants, in relation to reproduction, development and adaptation to the environment, but the sites of cell separation differ between species. Arabidopsis thaliana sheds individual floral organs (petals, sepals, and stamen) shortly after pollination [1], and displays opening of anthers and siliques (so-called dehiscence) to release pollen and drop seeds [2]. Dehiscence involves specialized tissues that breaks open when tension builds up upon dehydration [3]. The loss of organs is achieved by degradation of the middle lamella between adjacent cell files in specialized abscission zones (AZs) at the base of the organ to be shed. The abscission process can be divided in four stages, namely formation of the $\mathrm{AZ}$, conditioning of the $\mathrm{AZ}$ so it can respond to signaling molecules, cell wall loosening followed by the actual cell separation and finally generation of a protective layer [2]. In contrast to many other 
species, Arabidopsis does usually not shed cauline leaves, whole flowers, fruits or leaves; however, environmental stress factors like drought can induce abscission of cauline leaves [4]. Cell separation events are also found below ground, facilitating lateral root emergence and releasing root cap cells $[5,6]$.

During the last couple of decades the invention of technologies for genome-wide determination of gene expression levels, such as microarrays and, later, RNA sequencing, have facilitated a transcriptomic approach to understand these processes. This approach gives unbiased access to the gene expression pattern in a given organ at a given time. However, organs consist of several tissues, and such experiments do not disclose more specific expression patterns within an organ. Furthermore, genes with low expression levels, which is typical for signaling molecules, can easily be overshadowed by more abundantly expressed genes, which are not necessarily the most important factors with respect to control of whole processes. The genetic approach, that is, the identification of mutants impaired in the relevant process, can pick up important genes with low activity. From this perspective we review the evidence for the importance of the signaling peptide IDA and its receptors in different cell separation processes both above and below ground in Arabidopsis, and also in abscission of diverse organs in a variety flowering plants.

\section{The Role of IDA/IDL Peptides during Organ Abscission and Other Cell Separation Processes}

\subsection{Control of Cell Separation Processes by IDA and IDA-LIKE Peptides in Arabidospsis}

\subsubsection{Mutants Deficient in Abscission}

Few mutant blocking cell separation processes have been identified. The bop 1 bop 2 (BLADE-ON-PETIOLE1/2) double mutant in Arabidopsis and the jointless mutant in tomato fail to develop AZs at the base of the floral organs and at the base of the pedicel, respectively [7-9]. This indicates that organ abscission is dependent on specialized cells. Mutations in the Arabidopsis genes NEVERSHED (NEV) and INFLORESCENCE DEFICIENT IN ABSCISSION (IDA) result in permanently attached floral organs [10-12]. The nev mutant is impaired in the golgi apparatus, and most likely fail to export a number of proteins needed for middle lamella dissolution in the AZ [11].

IDA, on the other hand, is member of a small family of 9 genes encoding IDA and IDA-LIKE (IDL) preproproteins of less than 100 amino acids with an N-terminal export signal, a variable part, and near the C-terminus, a commonly conserved motif of 20 amino acids named the Extended PIP motif (EPIP) [10]. By analogy to CLAVATA3 (CLV3), one of the first signaling peptides identified, shown to be a ligand of a leucine-rich repeat (LRR) receptor-like kinase (RLK) (CLAVATA1), we anticipated that IDA could be a ligand of LRR-RLKs. Of the approximately 235 LRR-RLK genes in Arabidopsis, HAESA (HAE) and the closely related HAE-LIKE2 (HSL2), were found to be expressed in the floral organ AZs, and the hae hsl2 double mutant was, like ida, totally deficient in abscission (Supplementary Figure S1) [13,14].

The abscission process can be monitored by measuring the force needed to pull of the petals, the so-called petal breakstrength (pBS) [15]. In wild type Arabidopsis, the high pBS at anthesis is gradually reduced to zero due to cell wall loosening, and at position 8 (counting flowers from the top of the inflorescence) the organs are lost [13]. The ida and hae hsl2 mutants initially display a similar, but delayed pBS profile, thus indicating that IDA is regulating some cell wall remodelling (CWR) genes. For example, expression levels of XYLOGLUCAN ENDOTRANSGLYCOSYLASE6 (XTR6), POLYGALACTURONASE LATERAL ROOT (PGLR) and POLYGALACTURONASE ABSCISSION ZONE ARABIDOPSIS THALIANA (PGAZAT) are greatly reduced in ida and hae hsl 2 mutants during floral organ abscission [5], consistent with microarray and transcriptome data [16]. However after position 10 the pBS increases again to the level at anthesis [13].

In a recent reevaluation of the literature on the role of IDA in abscission it was suggested that the lack of detachment of the floral organs in these mutants was due to lowered turgor pressure resulting in failure of expansion of AZ cells [17]. However, the floral organs are in fact turgid when the pBS value is at its lowest (position 10), and normally remain like that at least up to position 16 
(Supplementary Figure S1). Furthermore, when exposed to ethylene the floral organs of both the ida mutant and wild type senesce, but only in the mutant the floral organs remain attached. Conversely, the nev mutant show deficiency in abscission although the AZ cells expand $[10,12]$. Thus, there is no strict correlation between turgor loss and failure to abscise, and also no strict correlation between cell expansion and abscission.

\subsubsection{IDA and Induction of Abscission}

The ability of IDA to induce abscission has been questioned [17]. However, overexpression of IDA, using the constitutive $35 \mathrm{~S}$ promoter, leads to earlier abscission of petals, sepals and filaments, as well as ectopic abscission at the base of the pedicels, branches and cauline leaves [13]. These are sites where abscission takes place in many other species, and in Arabidopsis addition of IDA is sufficient for induction of abscission. Thus, the other conditions and factors needed for abscission to take place must be present. Consistent with this, vestigial AZs have been discovered at these sites, and the HAE and HSL2 receptors are expressed there $[13,18]$. Three peptides with similarity to IDA, IDA-LIKE6 (IDL6), IDA-LIKE7 (IDL7) and IDA-LIKE8 (IDL8) (Supplementary Figure S2), are involved in biotic and abiotic stress defence [19], however, accumulating evidence suggests that signaling peptides can be involved both in development and defense [20]. Drought stress may, for example, induce IDA expression at the base of Arabidopsis cauline leaves, leading to abscission of these organs [4]. The fact that all cells are not falling apart from their neighboring cells in the 35S:IDA lines points to the requirement of conditioned cells that are responsive to IDA signaling. The 35S:IDA phenotype is dependent on functional HAE and HSL2 [13]. Biochemical and structural evidence has substantiated IDA as a ligand for HAE and HSL2 [21]. Activation of HSL2 results in an oxidative burst, and an assay where synthetic IDA peptides of different concentrations and lengths were added to tobacco leaf pieces expressing HSL2 demonstrated that the 12 amino acids of PIP with hydroxylated proline (Pro, $\mathrm{P}$ ) in position 7 could bind and activate the receptor efficiently at biologically relevant nanomolar concentrations, hundred times more efficiently than EPIP [21]. The histidine (His, $\mathrm{H}$ ) and asparagine (Asn, $\mathrm{N}$ ) in positions 11 and 12 seem essential for function, but the amino acids C-terminally to Asn can be removed.

Synthetic hydroxylated IDA PIP peptide (Figure 1) bound to the HAE-LRR was used to obtain the molecular structure of this ligand-receptor pair and their interaction with the co-receptor SOMATIC EMBRYOGENESIS RECEPTOR KINASE1 (SERK1) [22]. In vitro studies have shown that subtilases can digest IDA two amino acids upstream of PIP, suggesting that the peptide is 14, not 12 amino acids long [23]. So far, the active peptide present in planta has not been isolated. Although the expression patterns of IDL genes differ from that of IDA, overexpression of IDL1-5 resulted in phenotypes very similar to that of 35S:IDA [13]. This suggested that IDL peptides could signal through the receptors of IDA. However, quantity may overshadow low quality binding, as it turned out that only IDL1 could complement the ida mutation when under control of IDA's own promoter [13]. This indicates that the residues of particular functional importance are those that differ between IDA or IDL1 and IDL2-5, that is Pro position 4 and Arg position 10 which have been changed to alanine or serine (Ala, A; Ser, S), and lysine (Lys, K), respective (Figure 1). At present, receptors of IDL2-5 have not been identified.

Further dissection of the IDA gene showed that the variable part and the C-terminal end are not necessary for function, however, plants transformed with 35S:IDA constructs without the N-terminal export sequence do not display the overexpression phenotypes [13]. The prepropeptide is thus assumed to be exported to the endoplasmic reticulum, processed, and secreted to the apoplastic space where it can move and interact with its receptors present in the plasma membrane. 

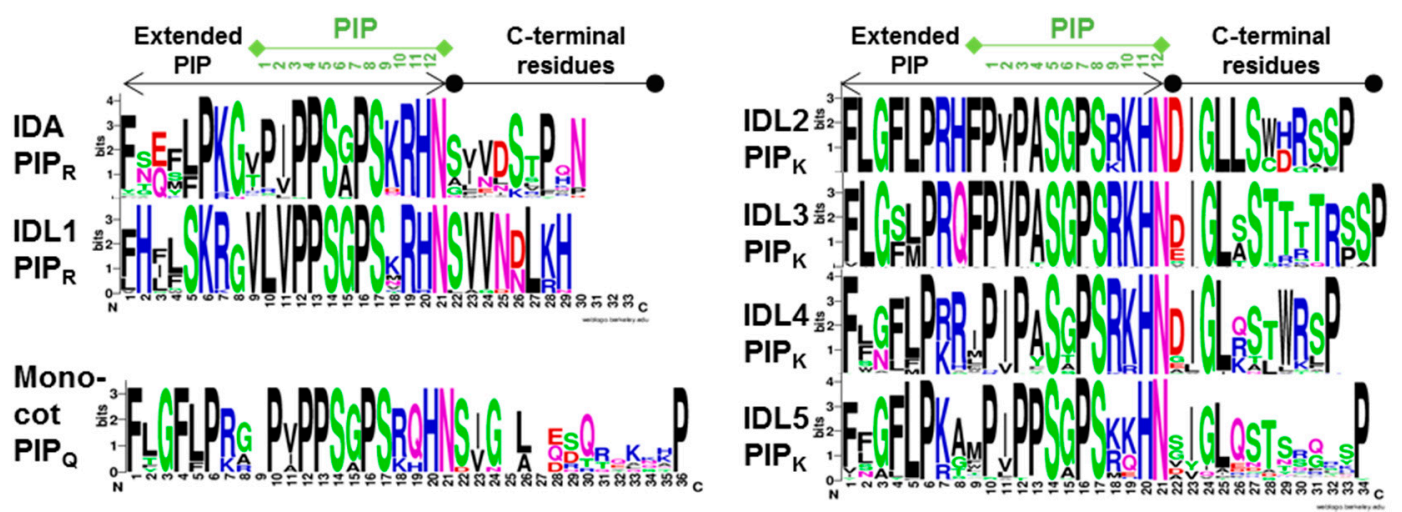

Figure 1. Conserved residues in propeptides of angiosperm IDA and IDL1-5 orthologues numbered from the start of the extended PIP motif to the C-terminal end. Sequences were aligned using Muscle, and consensus sequences were generated with logo@compbio.berkeley.edu. The PIP residues are numbered 1-12. IDL2-5 deviate from the IDA and IDL1 orthologues with a Lys (K) instead of an Arg (R) residue in position 10. IDL2, 3 and 4 have typically an Ala (A) instead of a Pro (P) in position 4. $\mathrm{PIP}_{\mathrm{Q}}$, which has a glutamine $(\mathrm{Gln} ; \mathrm{Q})$ in position 10 , was frequently found in the grasses (Poales) of the monocots [18].

\subsubsection{IDA and IDL1 Are Involved in Cell Separation Events in the Root}

We have discovered that IDA is also expressed below ground, in the cells overlying emerging lateral roots (LR) [5]. LRs are initiated in the pericycle in Arabidopsis; the LR primordia (LRP) have to pass three cell layers, endodermis, cortex and epidermis, to emerge (Figure 2). Mutations in IDA, HAE or HSL2 delay LR emergence, since the overlaying cells do not separate to let the lateral root primordia (LRP) out. However, these mutants do not stop the primordia from growing, and in ida and hae mechanical force from the growing LRP seems responsible for localized destruction of cells directly overlaying the emerging LRP [5]. Transcription factors working downstream of IDA-HAE/HSL2 during floral organ abscission and cell wall remodeling genes, typically expansins (EXPs), xyloglucan endotransglucosylase/hydrolases (XTHs), and PGs have been identified through suppressor screens and mutant studies [24,25]. Interestingly, a number of the same factors have been found to be involved in separation of cortex and epidermal cells to facilitate LR emergence $[5,26]$. 


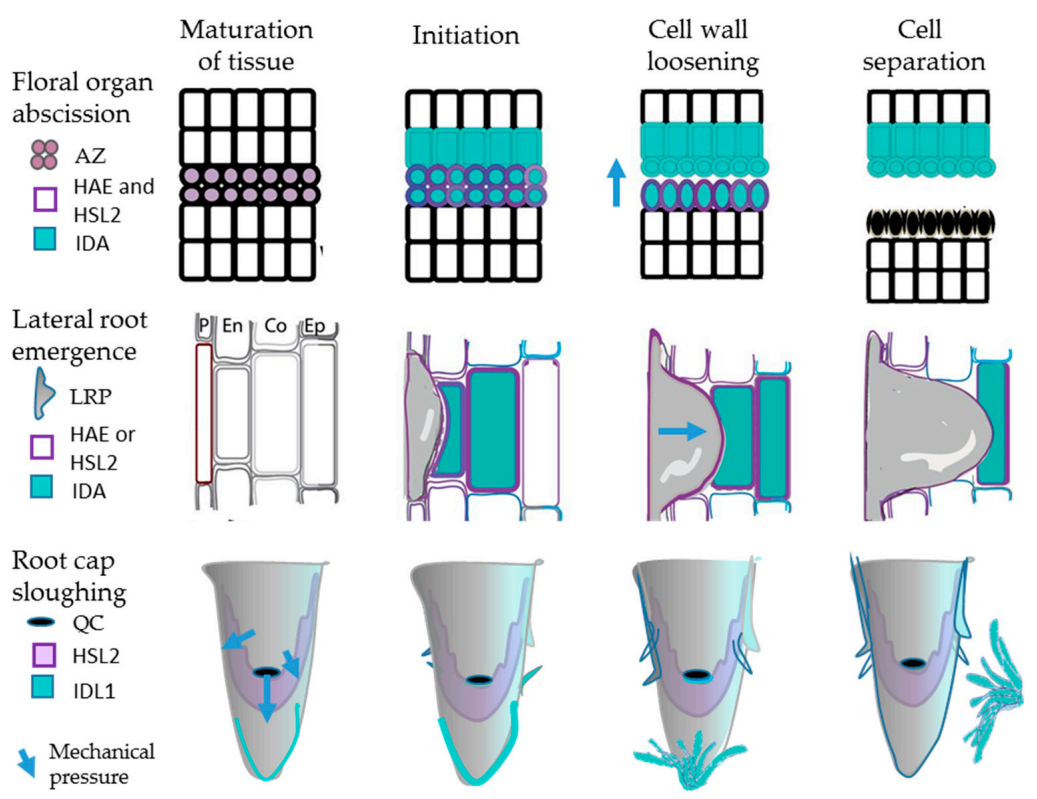

Figure 2. IDA/IDL and HAE/HSL2 involvement in floral organ abscission, lateral root emergence and root cap sloughing. These processes involve maturation of abscission zone (AZ) cells, cells overlying lateral root primordia (LRP), and lateral root cap (LRC) cells, respectively, and gradual cell wall loosening before the actual cell separation takes place. Mechanical pressure from expanded AZ cells in floral organ abscission, the LRP itself during emergence, and new LRC cells and elongating columella cells during root cap sloughing, is likely to contribute to these processes. QC-quiescent center.

Another cell separation event found in the Arabidopsis root is sloughing of root cap cell layers that cover the root tip with the meristematic zone, the stem cell niche surrounding the Quiescent Centre (QC) and the gravity-sensing columella cells [27] (Figure 2). IDL1 is expressed in the cap, which is protecting stem cells from damage and sensing environmental cues [13]. The outermost layer of the cap is detached at regular intervals. We have found that IDL1 and HSL2 are expressed at the tip, and, using the same methods as for IDA, it could be shown that IDL1 efficiently binds and activates HSL2 [6].

One might expect, in analogy with the floral mutant phenotype, that the idll and hsl2 mutants would accumulate root caps due to lack of cell separation. Instead the number of attached root cap layers was maintained, but the whole process, with maturation of columella and lateral root cap cells leading to the detachment of the outermost layer, and generation of new cell layers, was slowed down [6]. Enhanced expression levels of IDL1 generated more sloughed root caps, but the number of attached layers was still maintained. In contrast to other cell separation processes, root cap sloughing is a recurrent event in the same organ, and therefore it is necessary to compensate every sloughed layer by the generation of a new. In the mutants, the frequency of sloughing was significantly reduced, as fewer caps were detached.

From an evolutionary perspective, we find it interesting that seemingly very different developmental processes, above and below ground, employ the same molecular mechanism (Figure 2).

\subsection{Involvement of IDA/IDL1 in Abscission Processes in Other Flowering Plants}

\subsubsection{IDA/IDL1 and HAE/HSL Genes Are Present in All Angiosperm Orders}

We have undertaken a phylogenetic investigation to identify how widespread the IDA/IDL-HAE/HSL signaling module is in species other than Arabidopsis [18]. To investigate the evolutionary history of the IDA/IDL-HAE/HSL2 signaling module, we collected putative HAE/HSL2 and IDA/IDL sequences from The National Center of Biotechnology Information (NCBI), Phytozome and Comparative Genomics (GoCe), using the Basic Local Alignment Search Tool (BLASTp and tBLASTn). HSL2 orthologues were found in basal angiosperms, mono- and dicot orders, with the 
exception of the grasses (Poales), while HAE is the likely result of a whole-genome duplication in the dicot linage before the split into Rosids and Astrids [18].

We checked that potential IDA hits represented prepropeptides of about 100 amino acids with an N-terminal hydrophobic export signal and the PIP motif near the C-terminus. Analysis of the PIP motif in more detail identified three prototypes that were named after the position 10 residue, $P P_{R}$ representing IDA/IDL1, PIP $\mathrm{K}_{\mathrm{K}}$ representing IDL2-5, and in addition, $\mathrm{PIP}_{\mathrm{Q}}$ in particular found in the grasses (Poales) (Figure 1) [18]. We concluded that the $\mathrm{PIP}_{\mathrm{R}}$ motif has been conserved over the 175 million years flowering plants have evolved, as it was present in all orders from the most primitive to the most advanced angiosperms. Only two out of the twelve amino acids in the PIP $_{R}$ motif differ over evolutionary time, valine (Val, V) or isoleucine (Ile, I), which are found in position 2 and Ala or glycine (Gly, G) in position 6 . In both cases the alternative amino acids have similar properties. The $\mathrm{PIP}_{\mathrm{K}}$ variant could be followed back at least to the early eudicots [18]. IDL4, which, in addition to Lys10, is characterized by mutation in the Pro in position 4 (Figure 1), most likely arose after an early whole genome triplication before the appearance of the Vitis order [28].

Further analysis of the sequences collected revealed a correlation between the amino acid in position 10 and the amino acid motifs present on each side of the PIP motif, for example, the common amino acid C-terminally to PIP is Ser Val (SV) in IDA/IDL1 propeptids, but DIG in IDL2-5 (Figure 1). We assume that these are recognition sites for processing enzymes. There are 54 genes encoding subtilases in Arabidopsis and three of these, SBT4.12, SBT4.13 and SBT5.2 have been shown to process IDA between Lys and Gly in the conserved motif LPKG two amino acids upstream of PIP $P_{R}$ (Figure 1) [23]. The IDL propeptides have different signatures, suggesting that they are processed by other subtilases. Typically, each species has one or two genes encoding PIP ${ }_{\mathrm{R}}$ IDA peptides, and beside this, IDL4 PIP $\mathrm{K}$ variants. Some of these genes are the result of more recent genome duplications, like IDL1, IDL2 and IDL3, which are specific to Brassicae.

2.2.2. Expression Data and Functional Testing Suggest IDA/IDL Regulation of Abscission of Diverse Organs Both in Dicots and Monocots

To substantiate not only the presence of IDA/IDL and HAE/HSL genes in the genomes of flowering plants, but also their potential involvement in cell separation events, identification of relevant expression patterns is needed, such as expression in AZs of abscising organs (Table 1). Over the last few years when RNA sequencing and transcriptome analyses have become fast and economically affordable methods, gene expression patterns in AZs versus non-abscission zones have been investigated in many species, for example fruit trees like citrus species and litchi where abscission is a problem (Table 1). Such analyses reveal that similar CWR genes are transcribed, even when in different organs [29-33]. Compared to that of enzyme-encoding genes, transcription of signaling peptide genes may be less abundant and may therefore be overlooked, and additionally the focus has often been directed more towards traditional hormones.

One example is the recent transcriptome investigation of the AZs of yellow lupine (Lupinus luteus), belonging to legumes under the order Fabales of the Rosids. Lupine seeds are used as animal feed and a source for vegetative oil in parts of the world. Due to their high protein content, lupine seeds represent a significant alternative to soybean (Glycine max), but premature abscission of flowers is a problem that affects yield. 
Table 1. Overview of IDA/IDLs during abscission and other cell separation processes in plants.

\begin{tabular}{|c|c|c|c|}
\hline Genes & Process & Methods ${ }^{1}$ & Species \\
\hline IDA, HAE, HSL2 & Floral abscission & $\begin{array}{c}\text { Mutant; } \\
\text { 35S-phenotype; } \\
\text { Binding assay; } \\
\text { Crystal structure }\end{array}$ & Arabidopsis $[10,21,22,34]$ \\
\hline$I D A, H A E, H S L 2$ & LR emergence & Mutant & Arabidopsis [5] \\
\hline IDA, HAE, HSL2 & Leaf abscission & Mutant & Arabidopsis $[4,35]$ \\
\hline IDL1, HSL2 & Root cap sloughing & $\begin{array}{c}\text { Mutant; } \\
\text { Enhanced-phenotype; } \\
\text { Binding assay }\end{array}$ & Arabidopsis [6] \\
\hline $\begin{array}{c}\text { GmIDA2a/2b, } \\
\text { GmHAE3b/5a/5b }\end{array}$ & Leaf abscission & Expressed in AZ & Soybean [36] \\
\hline GmIDL2a/4b & LR emergence & 35S-phenotype & Soybean [37] \\
\hline LcIDL1 & Flower/Fruit abscission & $\begin{array}{c}\text { Expressed in AZ; } \\
\text { 35S-phenotype; } \\
\text { Rescue of } i d a \text { mutant }\end{array}$ & Litchi [38] \\
\hline CitIDA3 & Fruit abscission & $\begin{array}{c}\text { Expressed in AZ } \\
\text { 35S phenotype } \\
\text { Rescue of } i d a \text { mutant }\end{array}$ & Citrus [39] \\
\hline LIIDA & Flower abscission & $\begin{array}{l}\text { Expressed in AZ; } \\
\text { Synthetic peptide }\end{array}$ & Lupin [40] \\
\hline $\operatorname{EgIDA2/5}$ & Fruit abscission & $\begin{array}{l}\text { Expressed in AZ } \\
\text { Synthetic peptide }\end{array}$ & Oil palm $[18,41]$ \\
\hline PpIDA, PpIDL1 & Leaf abscission & $\begin{array}{l}\text { Expressed in AZ } \\
\text { Synthetic peptide }\end{array}$ & Poplar $[18,41]$ \\
\hline
\end{tabular}

The whole genome sequence of yellow lupine is not available, but RNA seq has been performed on $\mathrm{AZ}$ and non-AZ tissue, as well as AZ in flowers of different developmental stages, and transcriptional differences have been investigated with an explicit focus on cell wall modifications and hormone metabolism [31]. However, data on IDA, IDL and HSL transcript levels could be extracted from the project (BioProject ID PRJNA419564) by tBLASTn searches against the sequenced RNA using Arabidopsis HAE/HSL2 or the prepropeptides of IDA/IDL or as queries. Transcripts representing two putative lupin PIP R $_{\text {orthologues (named LIIDA and LiIDL1) and three PIP }}$ orthologues (LIIDL2, LlIDL3 and LlIDL4) as well as putative orthologues of HAE and HSL2 were identified (Figure 3a). LIIDA and LlHSL2 showed higher expression levels at the base of pedicels with rather than without developed AZ (Figure $3 b$ ), and the expression levels in AZs in developing flowers increased from green buds to the post-pollination stage (Figure $3 \mathrm{~b}, \mathrm{c}$ ). These data indicate that the lupine gene most similar to AtIDA plays the most important role in lupine AZs compared to the LIIDL genes, however, this has not been confirmed by other methods, such as qPCR.

The cDNA for one of the PIP $\mathrm{K}$ type genes with moderate expression levels compared to LIIDA, was recently cloned [40]. Application of 10-100 $\mu \mathrm{M}$ synthetic EPIP motif for this LIIDL gene on non-abscising AZ increased the number of abscised flowers. These concentrations are, however, comparable to overexpression using the $35 \mathrm{~S}$ promoter, and significantly higher than normal biological levels. When considering the published Arabidopsis data on the efficiency of different AtIDA/AtIDL peptides [21], it is not clear why an EPIP motif from an LlIDL gene and not a PIP $\mathrm{R}_{\mathrm{R}}$ motif from an LlIDA gene was chosen for this experiment.

Expression of IDL and HSL homologues have also been investigated in soybean, belonging to the order Fabales of the Rosids, which has four $\mathrm{PIP}_{\mathrm{R}}$ and eight $\mathrm{PIP}_{\mathrm{K}}$ genes [36]. In leaves, GmIDA2a and $2 b$ with ethylene-independent highest absolute and relative AZ expression level at the base of the petioles, are the paralogs most similar to AtIDA. Interestingly, GmHAE3b and GmHAE5a/5b with the highest similarities to AtHAE and AtHSL2, respectively, were also adequately expressed in the petiole [36]. Thus, these expression patterns suggest the involvement of the IDA-HAE/HSL2 module in leaf abscission. Interestingly, GmIDL2a, as well as the $\mathrm{PIP}_{\mathrm{K}}$ type GmIDL4b, are expressed in the cells 
overlaying lateral root primordia, and overexpression of the encoded peptides enhances LR emergence, ectopic separation of root cells, and increases the expression levels of EXPs, XTHs, and PGs [37].

The other major group of dicot plants, the Asterids, have, independently of the Rosids, gone through diverse whole genome duplications [28], but also have genes encoding $\mathrm{PIP}_{\mathrm{R}}$ and $\mathrm{PIP}_{\mathrm{K}}$ peptides, consistent with the identification of IDA and IDL4 homologues in eudicots that evolved before the Rosid-Asterid split. For instance in tomato (Solanum lycopersicum) there are one $\mathrm{PIP}_{\mathrm{K}}$ and several PIP genes, including one expressed in AZ [36].
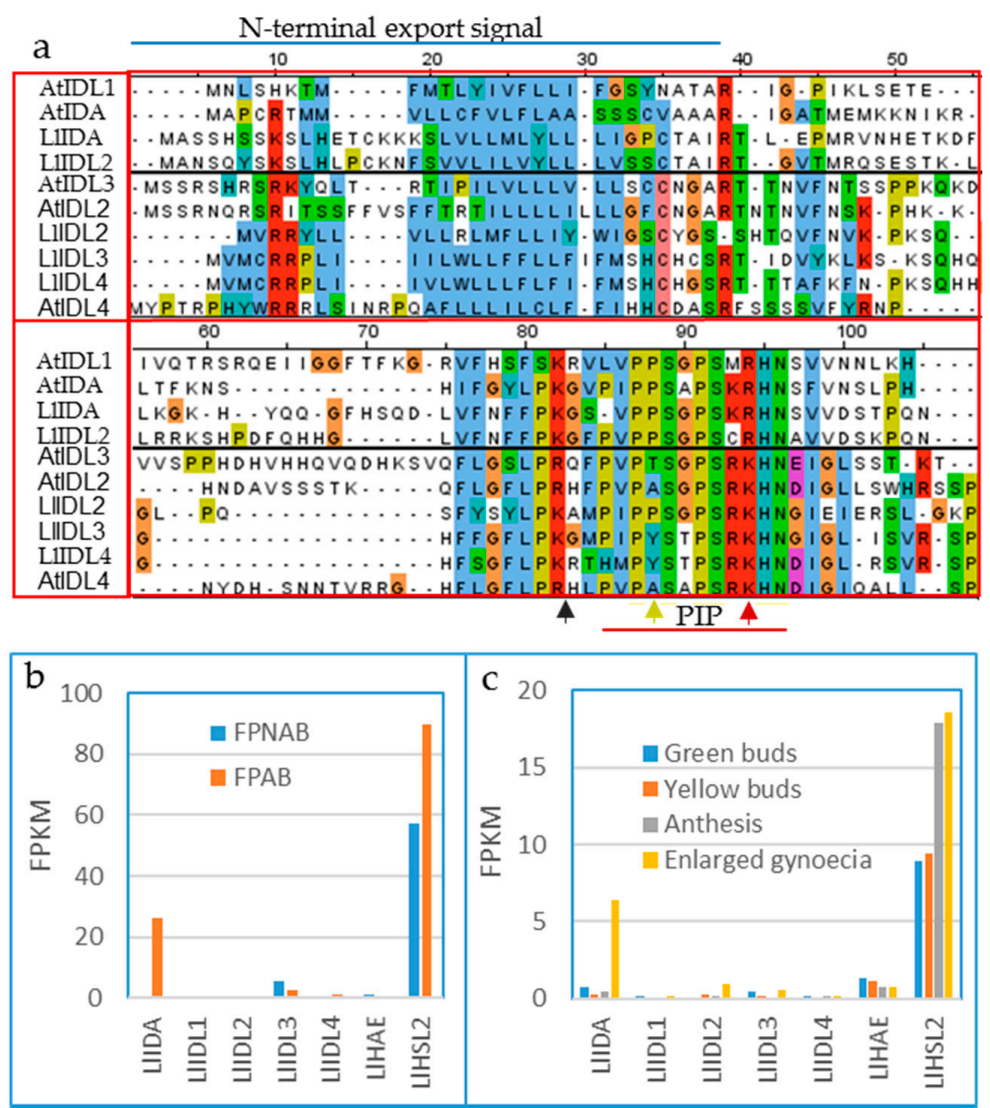

Figure 3. IDA-HAE/HSL2 of yellow lupine (Lupine luteus). (a) Multiple sequence alignment (generated with Jalview version 2) of prepropeptide sequences from Arabidopsis (AtIDA/AtIDL) and yellow lupine (LIIDA/LIIDL), colored with ClustalX color scheme (blue-hydrophobic; red-positive charge; mangenta—negative charge; green—polar; cyan—aromatic; cysteine—pink; proline-yellow; and glycine-orange). The N-terminal export signal and the PIP motif are indicated. Red arrow-position 10 of the PIP motif, either R or K; yellow arrow-position 4 of PIP, $\mathrm{P}$ often mutated in $\mathrm{PIP}_{\mathrm{K}}$ peptides; black arrow-suggested processing site for endopeptidase. Transcription level of LIIDA/LIIDL and LlHAE/LIHSL2 in (b) non-abscising (FPNAB) and abscising (FPAB) floral pedicels and (c) during four stages of flower development, identified by RNA sequencing (FPKM-Fragment Per Kilobase of exon per Million fragments mapped). Data was extracted from BioProject ID PRJNA419564). LIIDL1-OIV90565.1; LIIDL2-OIW07821.1; LIIDL3-OIW17824.1; LIIDL4-OIV98063.1; LIIDL5 AMH85930.1; LIIDA TRINITY_DN45322_c0_g2_i1, CP023114.1:17426727-17426888; LIHSL2-TRINITY_DN55300_c2_g2; LIHAE-TRINITY_DN57817_c0_g1.

We have chosen to investigate poplar, a species of the order Malpighiales, which is the most distant order of the Rosids clade compared to the Brassicales. Poplar trees grow in the northern hemisphere and shed their leaves in the autumn in response to lowered temperature and shorter day length. Experimentally, cell separation in the preformed AZ at the leaf axil of hybrid aspen (Populus 
tremula X Populus tremuloides), can be induced by wrapping branches in foil to keep the light out, thus mimicking the dark and long autumn nights [42]. A significant upregulation of PpIDA and PpIDL1 was seen in in these AZs [18].

Fall of whole flowers occurs both in monocot and eudicot species, when pollination or fertilization fail [43]. Abscission of immature fruit is a normal event in several cultivated crops [38,39]. For instance, in citrus (Citrus sinensis), of the order Sapindales of the Rosids, the expression of the gene encoding the peptide most similar to AtIDA (CitIDA3) could be correlated to the process of fruit abscission [39]. Interestingly, CitIDA3 promoter coupled to the GUS marker gene and transformed into Arabidopsis, was expressed in the floral organ AZs in flower positions 5-8 counting from the top of the inflorescence, the same positions where pAtIDA:GUS is expressed [39]. This suggests that genes expressed in the AZ cells of different organs in different species have conserved promoter elements recognized by conserved transcription factors. Interestingly, important downstream components of the IDA-HAE/HSL2 pathway, like the KNAT transcription factors and cell wall remodeling genes, have been identified in both Arabidopsis and Citrus [25].

Litchi (Litchi chinensis) is another fruit tree where abscission is a problem. From the first flowering there are waves of flower abscission after bloom. The loss is unpredictable, however, starting from 60,000 flowers per tree less than 50\% develop mature fruits. Three genes encoding IDA/IDL homologues have been identified, whereof the LcIDL1 expression level increases as the abscission process progresses in litchi floral AZs [38]. As for CitIDA3, the PIP motif of LcIDL1 is identical to that of Arabidopsis $\mathrm{PIP}_{\mathrm{R}}$ except for the Ala-Gly switch in position 6 . There is also a conservation of the flanking amino acid residues that are likely recognition sites for endopeptidases which process the preprotein to a mature protein. The peptides encoded by the two other LcIDL genes are more similar to AtIDL4 and AtIDL5, and their flanks differ, suggesting that they are processed by different endopeptidases.

A further indication of a conserved role of LcIDL1 in abscission was provided by rescue of the abscission deficient phenotype of the Arabidopsis ida mutant using LcIDL1 expressed under the promoter of AtIDA [38]. Additional substantiation of the function in cell separation was demonstrated by an increase in cytosolic $\mathrm{pH}$ in $\mathrm{AZ}$ cells, and induction of CWR genes previously shown to be regulated by IDA-HAE/HSL2 in Arabidopsis [38].

As for CitIDA3, 35S:LcIDL1 transformed into Arabidopsis resulted in a phenotype very similar to that of 35S:AtIDA, with early abscission [34,38,39]. However, the authors report that when using a 35S:LcIDL1-YFP construct transformed into protoplasts, the YFP appears in the cytosol. It is not expected that an IDA peptide can work in the cytosol, as the peptide must be transported to the apoplastic space to bind the ectodomain of the receptors. Therefore, we find it unlikely that the location of the YFP reflects the location of the active peptide. Instead, after processing on both sides of the PIP motif, the mature peptide could possibly be secreted while the C-terminal amino acids fused to YFP may remain in the cytosol. Alternatively, appropriate processing enzymes may not be expressed in leaf protoplasts.

Our phylogenetic investigation identified a $\mathrm{PIP}_{\mathrm{R}}$ version of the IDA homologues also in monocots, for example, in oil palm (Elaeis guineensis) of the order Arecales. Dicots and monocots diverged about $130 \mathrm{Myr}$ ago, and thus, the conservation of the peptide suggests a very strong natural selection against changes in the peptide sequence. We have reported an upregulation of EgIDA genes in ripened AZ of the oil palm fruits [18]. As in many other species, ethylene is produced at the onset of the abscission process, and contributes to the necessary maturation of the AZ cells [17,41]. In oil palm, ethylene is a prerequisite for abscission to take place and $E g I D A 5$ is induced by exogenous ethylene. AtIDA was launched as a factor representing an ethylene-independent pathway of abscission in the sense that the ida mutant phenotype cannot be rescued by exposure to ethylene, and does not display phenotypes, for example, hypocotyl elongation of dark-grown seedlings, typical for mutations in genes involved in perception of ethylene [10]. However, in Arabidopsis, ethylene is also produced in mature AZ when AtIDA is expressed [44]. 


\subsubsection{Matching Ligands and Receptors}

Our phylogenetic investigation has also addressed the ancestry of the receptors HAE and HSL2 [18]. With the exception of the grasses, receptors most similar to HSL2 were found present in all orders investigated, indicating that an orthologue of HSL2 existed already when the flowering plants emerged. HAE is, on the other hand, the likely result of a genome duplication that took place in early dicots [18]. The upregulated expression of IDA genes in the AZ in several species, albeit in different organs, is consistent with a conserved function in regulation of cell separation processes. This requires AZ expression of receptors that are conserved both regarding downstream output from the kinase domain upon receptor activation and with respect to peptide and co-receptor binding. SERK1, a co-receptor identified in all land plants [45], in Arabidopsis interacts with IDA and HAE [22], and SERK1-interacting residues, Arg-His-Asn (RHN), at the C-terminal end of PIP $_{R}$ as well as the amino acids involved in co-receptor binding in HAE/HSL2, are also nicely conserved in the receptors discussed here (Supplementary Figure S3).

The crystal structures of AtIDA and AtIDL1 synthetic peptides bound to AtHAE have recently been solved, and revealed that the ligand is positioned along the inner surface of the LRR ectodomain of the receptor [22]. The residues directly interacting with the amino acids of the peptide are among the best conserved, apart from the leucine (Leu, L), Asn, Gly, isoleucine (Ile, I) and Pro residues that make up the prototype scaffold of the 24 amino acid long LRR units (LxxLxxLxLxxNxLSGxIPxxLGx where $x$ is any amino acid). Using the AtHAE-AtIDA crystal structure (PDBid:5ixq) [22] and homology modelling (SWISS-model) [46], three-dimensional structures were generated for the ectodomain of AtHSL2, and the HSL2 othologue from E. guineensis (EgHSL2). [18]. The interactions between these modelled receptors and the respective IDA peptides were investigated. Consistent with binding and activation data [21], there is a high degree of conservation between AtHAE and AtHSL2, likely to facilitate strong interaction between AtIDA and the AtHSL2 receptor (Figure 4a), and most peptide-receptors hydrogen bonds seem to be preserved (Figure $4 \mathrm{~b}$ ). The $\mathrm{COO}^{-}$group of Asn in the AtIDA peptide forms hydrogen bonds with Arg407 and Arg409 in AtHAE [22]. However, Arg407 is replaced with Tyr415 in AtHSL2, likely weakening the interaction with the C-terminal Arg-His-Asn motif in AtIDA. Nonetheless, the hydrogen bonding network in the vicinity of the central hydroxylated Pro (Hyp) of AtIDA is likely strengthened in the AtHSL2-AtIDA model, compared to in the AtHAE-AtIDA crystal structure, due to the presence of an Asn residue (Asn296) in AtHSL2, replacing Arg288 in AtHAE (Figure 4a,b).

The overall interaction between the modelled EgHSL2 receptor with the corresponding EgIDA peptide (Figure 4c) seems to be weakened compared to the interactions seen for the Arabidopsis AtHAE-AtIDA crystal structure and AtHSL2 model. It is, however, still likely to be sufficient for a receptor-peptide binding interaction (Figure 4d) if the central Hyp is replaced with a non-hydroxylated proline in the EgIDA peptide. A weaker hydrogen bonding network in this region is likely in this species, although a probable hydrogen bond is formed between the nearby Trp272 residue in EgHSL2 (Phe268 AtHAE) and the carbonyl group of Gly residue in EgIDA, strengthening the interaction with the peptide. 
a

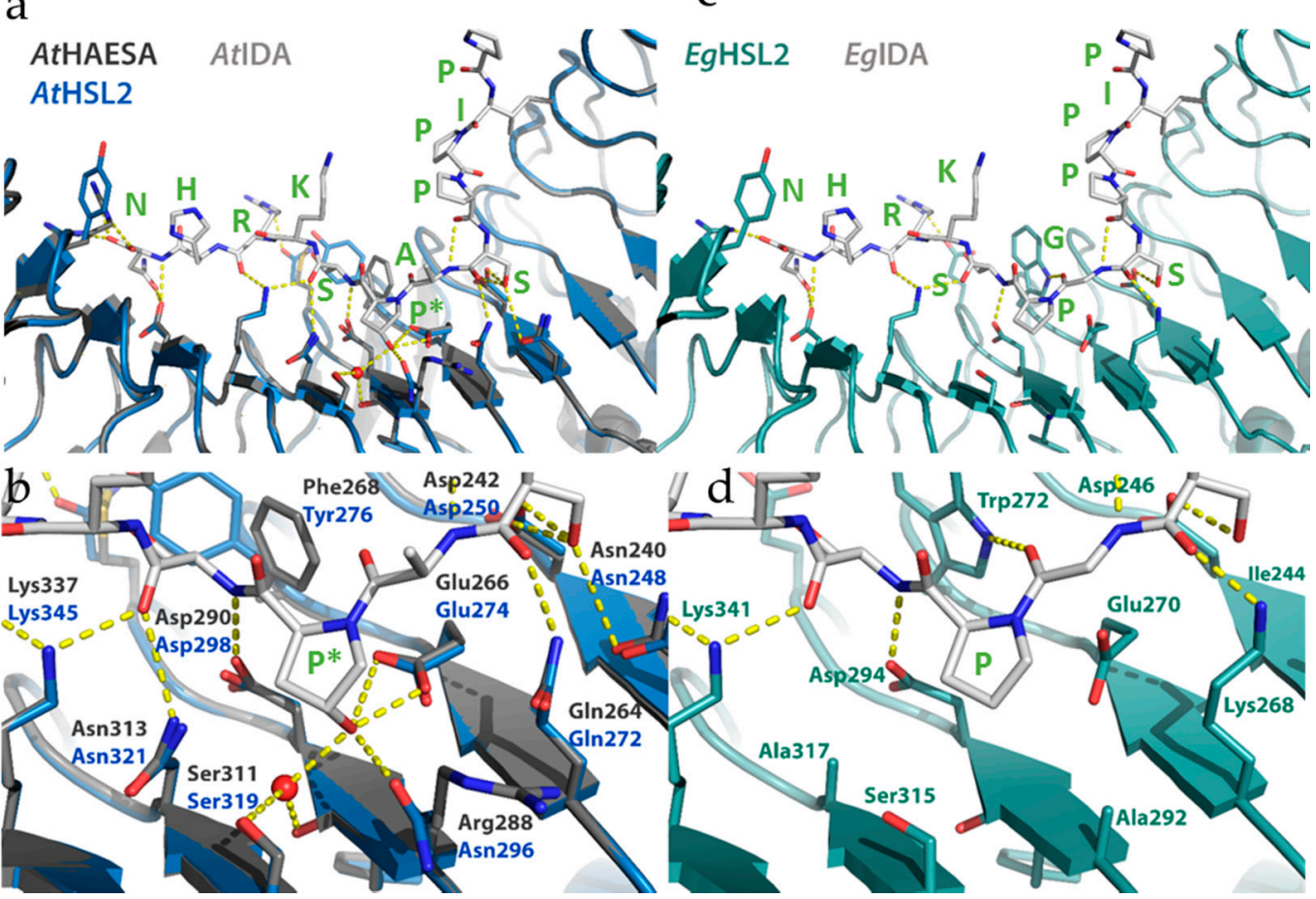

Figure 4. Structures of AtHAE, AtHSL2, and EgHSL2, with bound AtIDA and EgIDA peptides. (a) The AtIDA binding pocket covers leucine-rich repeats (LRRs) 2-14 and all residues originate from the inner surface of the AtHAE superhelix. Overall alignment of the AtHAE structure (PDBid:5ixq) (dark grey) with the AtHSL2 model (blue), with AtIDA peptide bound (light grey), with (b) close-up view of the central Hypregion. (c) Overall model of EgHSL2 (teal) with EgIDA peptide (light grey). with (d) close-up view of the latter structure and models showing the central parts in EgHSL2 with the surrounding hydrogen bonding network. Central amino acids of the receptors, as well as the IDA peptides, are shown as sticks and colored by atom type. Water molecules are shown as red spheres. Hydrogen bonds are depicted as dotted lines (yellow). Residues involved in hydrogen bonding to the peptide are depicted with three-letter symbols in colors according to the respective structures, as in $(\mathbf{a}, \mathbf{c})$. The peptide residues are shown in green wone-letter symbols. $\mathrm{P}^{*}$-hydoxylated Pro. All structure figures were prepared using PyMOL (Schrödinger, LLC).

To investigate the interaction between the EgHSL2 receptor and the corresponding EgSERK1 co-receptor, a homology model of the EgHSL2-EgIDA-EgSERK1 signaling complex was generated (SWISS-model), using the Arabidopsis AtHAE-AtIDA-AtSERK1 complex structure (PDBid:5iyx) as a template [22]. A structural alignment of the model and structure is shown in Figure 5. Residues on the SERK1 co-receptor, previously shown to be involved in hydrogen bonding to the HAE receptor, are conserved in AtSERK1 and EgSERK1 (Supplementary Figure S3). Also, a high degree of conservation is shown in the HAE/HSL2-SERK1 interface in the E. guineensis model complex, as compared to the $A$. thaliana crystal complex. As also observed from the EgHSL2-EgIDA interaction (Figure 5), although fewer hydrogen bonds are suggested between EgHSL2 and EgSERK1 from the predicted EgHSL2-EgSERK1 model, the interaction is still likely sufficient for complex formation. 

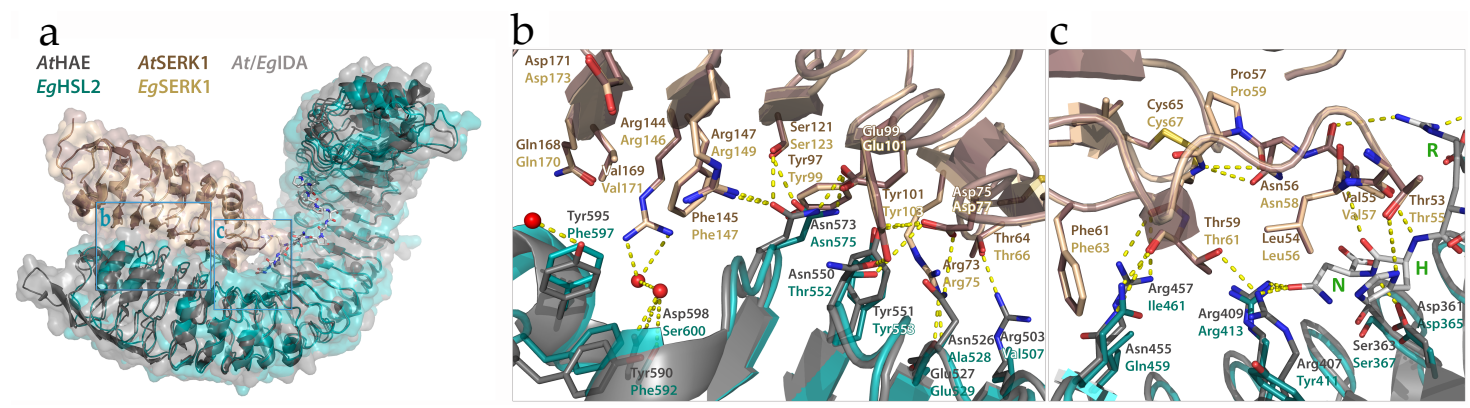

Figure 5. Structural alignment of the of $A$. thaliana HAE-IDA-SERK1 crystal complex (PDBid:5iyx) and the E. guineensis HSL2-IDA-SERK1 model complex. (a) Overall alignment of the two structures in cartoon and surface representation, with IDA shown as sticks. (b) Alignment of the hydrogen bonding network in the HAE/HSL2-SERK1 core interface and (c) in the HAE/HSL2-IDA-SERK1 interface in both complexes. Central amino acids, as well as the IDA peptides, are shown as sticks and colored by atom type. Water molecules are shown as red spheres. Hydrogen bonds are depicted as dotted lines (yellow). Residues involved in hydrogen bonding are depicted in colors according to the respective structures, as specified in (a). All structure figures were prepared using PyMOL (Schrödinger, LLC).

\section{Conclusions and Perspectives}

Starting with the identification of the different components of the IDA-HSL signaling pathway in Arabidopsis, we and others have now identified orthologues of these genes in all orders of flowering plants; provided examples of their expression in AZs or flowers, leaves and fruits; demonstrated that IDA peptide can induce the abscission processes both in monocots and dicots; and in a few cases validated the hypothesized conserved function across organ and species by complementation of the Arabidopsis ida mutant. The ultimate proof of conserved function is however still lacking-a total deficiency in abscission by mutation of the peptide or receptor genes has so far only been shown in Arabidopsis.

Our lab initiated the study of the ida mutant about 20 years ago due to its distinct abscission-deficient phenotype. Over these years technological innovations have revolutionized science, including plant science, so, in principle, we can access the genetic information of any species, any individual and even any cell. Whole genome sequencing of species from every order of flowering plants makes it possible to study variation in morphology, development and environmental adaptation from an evolutionary perspective.

Peptides have been discovered as novel hormones facilitating cell-to-cell communication both in relation to developmental processes and defense (see recent reviews [47-50]). IDA-HAE/HSL2 is among the best elaborated peptide-ligand receptor modules per date, with genetic, biochemical and structural evidence for peptide-receptor interaction, identification of co-receptors, and components of a downstream signaling pathway with MAP kinases and KNOX transcription factors, controlling the expression of genes involved in the actual cell-separation step $[4-6,10,13,14,21,23,25,26,34,35]$. One fundamental question in evolutionary biology is whether evolution of traits is mainly a result of mutational changes in proteins or changes in the regulation of when and where genes are expressed. We hypothesize that with respect to abscission processes the latter is the case; the molecular basis for cell separation is conserved in the IDA-HSL ligand receptor signaling module no matter where and when organ abscission takes place.

Farmers suffer severe losses due to untimely abscission of flowers and fruits, and higher yield per acre would also be welcome from an environmental perspective. With the adaptation, in a steadily increasing number of plants species, of the CRISP/Cas gene editing technique that promises targeted mutagenesis of any gene, we anticipate that our hypothesis will be tested by generation of IDA or HSL mutants in relevant species in the near future. 
Supplementary Materials: The following are available online at http://www.mdpi.com/2223-7747/8/7/225/s1, Figure S1: The double mutant hae hsl2 is, like the ida mutant, totally deficient in abscission, Figure S2: Orthologous angiosperm sequences IDL6-8, Figure S3: Structure-based sequence alignment of the 21 leucine-rich repeats (LRRs) comparing Arabidopsis HAE and HSL2 with EgHSL2 from oil palm.

Author Contributions: C.-L.S. revised and completed the text and the figures; R.M.A. provided sequences, alignments and logos for the IDA and IDL peptides; M.H. generated and analysed the structural models; and R.B.A. wrote the paper.

Funding: This research received no external funding.

Acknowledgments: We thank Paulina Glazińska for assistance with the IDL and HSL sequences of L. luteus.

Conflicts of Interest: The authors declare no conflict of interest.

\section{References}

1. Bleecker, A.; Patterson, S.E. Last exit: Senescence, abscission, and meristem arrest in Arabidopsis. Plant Cell 1997, 9, 1169-1179. [CrossRef] [PubMed]

2. Patterson, S.E. Cutting Loose. Abscission and Dehisence in Arabidopsis. Plant Physiol. 2001, 126, 494-500. [CrossRef] [PubMed]

3. Patharkar, O.R.; Walker, J.C. Connections between abscission, dehiscence, pathogen defense, drought tolerance, and senescence. Plant Sci. Int. J. Exp. Plant Boil. 2019, 284, 25-29. [CrossRef] [PubMed]

4. Patharkar, O.R.; Walker, J.C. Core Mechanisms Regulating Developmentally Timed and Environmentally Triggered Abscission. Plant Physiol. 2016, 172, 510-520. [CrossRef] [PubMed]

5. Kumpf, R.P.; Shi, C.L.; Larrieu, A.; Sto, I.M.; Butenko, M.A.; Peret, B.; Riiser, E.S.; Bennett, M.J.; Aalen, R.B. Floral organ abscission peptide IDA and its HAE/HSL2 receptors control cell separation during lateral root emergence. Proc. Natl. Acad. Sci. USA 2013, 110. [CrossRef] [PubMed]

6. Shi, C.-L.; von Wangenheim, D.; Herrmann, U.; Wildhagen, M.; Kulik, I.; Kopf, A.; Ishida, T.; Olsson, V.; Anker, M.K.; Albert, M.; et al. The dynamics of root cap sloughing in Arabidopsis is regulated by peptide signalling. Nat. Plants 2018, 4, 596-604. [CrossRef] [PubMed]

7. Mao, L.; Begum, D.; Chuang, H.W.; Budiman, M.A.; Szymkowiak, E.J.; Irish, E.E.; Wing, R.A. JOINTLESS is a MADS-box gene controlling tomato flower abscission zone development. Nature 2000, 406, 910-913. [CrossRef] [PubMed]

8. McKim, S.M.; Stenvik, G.E.; Butenko, M.A.; Kristiansen, W.; Cho, S.K.; Hepworth, S.R.; Aalen, R.B.; Haughn, G.W. The BLADE-ON-PETIOLE genes are essential for abscission zone formation in Arabidopsis. Development 2008, 135, 1537-1546. [CrossRef] [PubMed]

9. Hepworth, S.R.; Zhang, Y.; McKim, S.; Li, X.; Haughn, G.W. BLADE-ON-PETIOLE-dependent signaling controls leaf and floral pattering in Arabidpsis. Plant Cell 2005, 17, 1434-1448. [CrossRef]

10. Butenko, M.A.; Patterson, S.E.; Grini, P.E.; Stenvik, G.-E.; Amundsen, S.S.; Mandal, A.; Aalen, R.B. INFLORESCENCE DEFICIENT IN ABSCISSION Controls Floral Organ Abscission in Arabidopsis and Identifies a Novel Family of Putative Ligands in Plants. Plant Cell 2003, 15, 2296-2307. [CrossRef]

11. Liljegren, S.J.; Leslie, M.E.; Darnielle, L.; Lewis, M.W.; Taylor, S.M.; Luo, R.; Geldner, N.; Chory, J.; Randazzo, P.A.; Yanofsky, M.F.; et al. Regulation of membrane trafficking and organ separation by the NEVERSHED ARF-GAP protein. Development 2009, 136, 1909-1918. [CrossRef] [PubMed]

12. Liu, B.; Butenko, M.A.; Shi, C.-L.; Bolivar, J.L.; Winge, P.; Stenvik, G.-E.; Vie, A.K.; Leslie, M.E.; Brembu, T.; Kristiansen, W.; et al. NEVERSHED and INFLORESCENCE DEFICIENT IN ABSCISSION are differentially required for cell expansion and cell separation during floral organ abscission in Arabidopsis thaliana. J. Exp. Bot. 2013, 64. [CrossRef] [PubMed]

13. Stenvik, G.E.; Tandstad, N.M.; Guo, Y.; Shi, C.L.; Kristiansen, W.; Holmgren, A.; Clark, S.E.; Aalen, R.B.; Butenko, M.A. The EPIP peptide of INFLORESCENCE DEFICIENT IN ABSCISSION is sufficient to induce abscission in arabidopsis through the receptor-like kinases HAESA and HAESA-LIKE2. Plant Cell 2008, 20, 1805-1817. [CrossRef] [PubMed]

14. Cho, S.K.; Larue, C.T.; Chevalier, D.; Wang, H.; Jinn, T.L.; Zhang, S.; Walker, J.C. Regulation of floral organ abscission in Arabidopsis thaliana. Proc. Natl. Acad. Sci. USA 2008, 105, 15629-15634. [CrossRef] [PubMed] 
15. Shi, C.-L.; Butenko, M.A. Quantitative Analysis of Floral Organ Abscission in Arabidopsis Via a Petal Breakstrength Assay. In Plant Senescence: Methods and Protocols; Guo, Y., Ed.; Springer: New York, NY, USA, 2018; pp. 81-88. [CrossRef]

16. Cai, S.; Lashbrook, C.C. Stamen abscission zone transcriptome profiling reveals new candidates for abscission control: Enhanced retention of floral organs in transgenic plants overexpressing Arabidopsis ZINC FINGER PROTEIN2. Plant Physiol. 2008, 146, 1305-1321. [CrossRef] [PubMed]

17. Meir, S.; Philosoph-Hadas, S.; Riov, J.; Tucker, M.L.; Patterson, S.E.; Roberts, J.A. Re-evaluation of the ethylene-dependent and -independent pathways in the regulation of floral and organ abscission. J. Exp. Bot. 2019, 70, 1461-1467. [CrossRef]

18. Stø, I.M.; Orr, R.J.S.; Fooyontphanich, K.; Jin, X.; Knutsen, J.M.B.; Fischer, U.; Tranbarger, T.J.; Nordal, I.; Aalen, R.B. Conservation of the abscission signaling peptide IDA during Angiosperm evolution: Withstanding genome duplications and gain and loss of the receptors HAE/HSL2. Front. Plant Sci. 2015, 6. [CrossRef]

19. Vie, A.K.; Najafi, J.; Winge, P.; Cattan, E.; Wrzaczek, M.; Kangasjarvi, J.; Miller, G.; Brembu, T.; Bones, A.M. The IDA-LIKE peptides IDL6 and IDL7 are negative modulators of stress responses in Arabidopsis thaliana. J. Exp. Bot. 2017, 68, 3557-3571. [CrossRef]

20. Wang, X.; Hou, S.; Wu, Q.; Lin, M.; Acharya, B.R.; Wu, D.; Zhang, W. IDL6-HAE/HSL2 impacts pectin degradation and resistance to Pseudomonas syringae pv tomato DC3000 in Arabidopsis leaves. Plant J. Cell Mol. Boil. 2017, 89, 250-263. [CrossRef]

21. Butenko, M.A.; Wildhagen, M.; Albert, M.; Jehle, A.; Kalbacher, H.; Aalen, R.B.; Felix, G. Tools and Strategies to Match Peptide-Ligand Receptor Pairs. Plant Cell 2014, 26, 1838-1847. [CrossRef]

22. Santiago, J.; Brandt, B.; Wildhagen, M.; Hohmann, U.; Hothorn, L.A.; Butenko, M.A.; Hothorn, M. Mechanistic insight into a peptide hormone signaling complex mediating floral organ abscission. eLife 2016, 5, e15075. [CrossRef]

23. Schardon, K.; Hohl, M.; Graff, L.; Pfannstiel, J.; Schulze, W.; Stintzi, A.; Schaller, A. Precursor processing for plant peptide hormone maturation by subtilisin-like serine proteinases. Science 2016, 354, 1594-1597. [CrossRef] [PubMed]

24. Niederhuth, C.; Patharkar, O.R.; Walker, J. Transcriptional profiling of the Arabidopsis abscission mutant hae hsl2 by RNA-Seq. BMC Genom. 2013, 14, 37. [CrossRef]

25. Shi, C.-L.; Stenvik, G.-E.; Vie, A.K.; Bones, A.M.; Pautot, V.; Proveniers, M.; Aalen, R.B.; Butenko, M.A. Arabidopsis Class I KNOTTED-Like Homeobox Proteins Act Downstream in the IDA-HAE/HSL2 Floral Abscission Signaling Pathway. Plant Cell Online 2011, 23, 2553-2567. [CrossRef] [PubMed]

26. Zhu, Q.; Shao, Y.; Ge, S.; Zhang, M.; Zhang, T.; Hu, X.; Liu, Y.; Walker, J.; Zhang, S.; Xu, J. A MAPK cascade downstream of IDA-HAE/HSL2 ligand-receptor pair in lateral root emergence. Nat. Plants 2019, 5, 414-423. [CrossRef]

27. Kumpf, R.P.; Nowack, M.K. The root cap: A short story of life and death. J. Exp. Bot. 2015, 66, 5651-5662. [CrossRef]

28. Tang, H.; Wang, X.; Bowers, J.E.; Ming, R.; Alam, M.; Paterson, A.H. Unraveling ancient hexaploidy through multiply-aligned angiosperm gene maps. Genome Res. 2008, 18, 1944-1954. [CrossRef]

29. Kim, J.; Yang, J.; Yang, R.; Sicher, R.C.; Chang, C.; Tucker, M.L. Transcriptome Analysis of Soybean Leaf Abscission Identifies Transcriptional Regulators of Organ Polarity and Cell Fate. Front. Plant Sci. 2016, 7, 125. [CrossRef]

30. Lee, Y.; Yoon, T.H.; Lee, J.; Jeon, S.Y.; Lee, J.H.; Lee, M.K.; Chen, H.; Yun, J.; Oh, S.Y.; Wen, X.; et al. A Lignin Molecular Brace Controls Precision Processing of Cell Walls Critical for Surface Integrity in Arabidopsis. Cell 2018, 173, 1468-1480.e9. [CrossRef]

31. Glazinska, P.; Wojciechowski, W.; Kulasek, M.; Glinkowski, W.; Marciniak, K.; Klajn, N.; Kesy, J.; Kopcewicz, J. De novo Transcriptome Profiling of Flowers, Flower Pedicels and Pods of Lupinus luteus (Yellow Lupine) Reveals Complex Expression Changes during Organ Abscission. Front. Plant Sci. 2017, 8, 641. [CrossRef]

32. Kim, J.; Sundaresan, S.; Philosoph-Hadas, S.; Yang, R.; Meir, S.; Tucker, M.L. Examination of the Abscission-Associated Transcriptomes for Soybean, Tomato, and Arabidopsis Highlights the Conserved Biosynthesis of an Extensible Extracellular Matrix and Boundary Layer. Front. Plant Sci. 2015, 6. [CrossRef] [PubMed] 
33. Sundaresan, S.; Philosoph-Hadas, S.; Riov, J.; Belausov, E.; Kochanek, B.; Tucker, M.L.; Meir, S. Abscission of flowers and floral organs is closely associated with alkalization of the cytosol in abscission zone cells. J. Exp. Bot. 2015, 66, 1355-1368. [CrossRef] [PubMed]

34. Stenvik, G.E.; Butenko, M.A.; Urbanowicz, B.R.; Rose, J.K.; Aalen, R.B. Overexpression of INFLORESCENCE DEFICIENT IN ABSCISSION activates cell separation in vestigial abscission zones in Arabidopsis. Plant Cell 2006, 18, 1467-1476. [CrossRef] [PubMed]

35. Patharkar, O.R.; Gassmann, W.; Walker, J.C. Leaf shedding as an anti-bacterial defense in Arabidopsis cauline leaves. PLoS Genet. 2017, 13, e1007132. [CrossRef] [PubMed]

36. Tucker, M.L.; Yang, R. IDA-like gene expression in soybean and tomato leaf abscission and requirement for a diffusible stelar abscission signal. AoB Plants 2012, 2012, pls035. [CrossRef] [PubMed]

37. Liu, C.; Zhang, C.; Fan, M.; Ma, W.; Chen, M.; Cai, F.; Liu, K.; Lin, F. GmIDL2a and GmIDL4a, Encoding the Inflorescence Deficient in Abscission-Like Protein, Are Involved in Soybean Cell Wall Degradation during Lateral Root Emergence. Int. J. Mol. Sci. 2018, 19, 2262. [CrossRef] [PubMed]

38. Ying, P.; Li, C.; Liu, X.; Xia, R.; Zhao, M.; Li, J. Identification and molecular characterization of an IDA-like gene from litchi, LcIDL1, whose ectopic expression promotes floral organ abscission in Arabidopsis. Sci. Rep. 2016, 6, 37135. [CrossRef] [PubMed]

39. Estornell, L.H.; Wildhagen, M.; Perez-Amador, M.A.; Talon, M.; Tadeo, F.R.; Butenko, M.A. The IDA Peptide Controls Abscission in Arabidopsis and Citrus. Front. Plant Sci. 2015, 6, 1003. [CrossRef]

40. Wilmowicz, E.; Kućko, A.; Ostrowski, M.; Panek, K. INFLORESCENCE DEFICIENT IN ABSCISSION-like is an abscission-associated and phytohormone-regulated gene in flower separation of Lupinus luteus. Plant Growth Regul. 2018, 85, 91-100. [CrossRef]

41. Tranbarger, T.J.; Domonhedo, H.; Cazemajor, M.; Dubreuil, C.; Fischer, U.; Morcillo, F. The PIP Peptide of INFLORESCENCE DEFICIENT IN ABSCISSION Enhances Populus Leaf and Elaeis guineensis Fruit Abscission. Plants 2019, 8, 143. [CrossRef]

42. Jin, X.; Zimmermann, J.; Polle, A.; Fischer, U. Auxin is a long-range signal that acts independently of ethylene signaling on leaf abscission in Populus. Front. Plant Sci. 2015, 6, 634. [CrossRef] [PubMed]

43. Van Doorn, W.G. Effect of ethylene on flower abscission: A survey. Ann. Bot. (Lond.) 2002, 89, 689-693. [CrossRef] [PubMed]

44. Butenko, M.A.; Stenvik, G.E.; Alm, V.; Saether, B.; Patterson, S.E.; Aalen, R.B. Ethylene-dependent and -independent pathways controlling floral abscission are revealed to converge using promoter:reporter gene constructs in the ida abscission mutant. J. Exp. Bot. 2006, 57, 3627-3637. [CrossRef] [PubMed]

45. Aan den Toorn, M.; Albrecht, C.; de Vries, S. On the Origin of SERKs: Bioinformatics Analysis of the Somatic Embryogenesis Receptor Kinases. Mol. Plant 2015, 8, 762-782. [CrossRef] [PubMed]

46. Arnold, K.; Bordoli, L.; Kopp, J.; Schwede, T. The SWISS-MODEL workspace: A web-based environment for protein structure homology modelling. Bioinformatics 2005, 22, 195-201. [CrossRef] [PubMed]

47. Olsson, V.; Joos, L.; Zhu, S.; Gevaert, K.; Butenko, M.A.; De Smet, I. Look Closely, the Beautiful May Be Small: Precursor-Derived Peptides in Plants. Annu. Rev. Plant Biol. 2019, 70, 153-186. [CrossRef] [PubMed]

48. Hirakawa, Y.; Sawa, S. Diverse function of plant peptide hormones in local signaling and development. Curr. Opin. Plant Biol. 2019, 51, 81-87. [CrossRef] [PubMed]

49. Bartels, S.; Boller, T. Quo vadis, Pep? Plant elicitor peptides at the crossroads of immunity, stress, and development. J. Exp. Bot. 2015, 66, 5183-5193. [CrossRef] [PubMed]

50. Albert, M. Peptides as triggers of plant defence. J. Exp. Bot. 2013, 64, 5269-5279. [CrossRef] [PubMed]

(C) 2019 by the authors. Licensee MDPI, Basel, Switzerland. This article is an open access article distributed under the terms and conditions of the Creative Commons Attribution (CC BY) license (http://creativecommons.org/licenses/by/4.0/). 